\title{
Modeling of Split Ring Resonators Loaded Microstrip Line with Different Orientations
}

\author{
Rajni*, Gurwinder Singh**, Anupma Marwaha*** \\ *,** Department of Electronics \& Communication Engineering, Shaheed Bhagat Singh State Technical Campus, \\ Ferozepur, Punjab, India \\ *** Department of Electronics \& Communication Engineering, Sant Longowal Institute of Engg. \& Technology, \\ Longowal (Sangrur), Punjab, India
}

\begin{tabular}{|c|c|}
\hline Article Info & ABSTRACT \\
\hline Article history: & \multirow{8}{*}{$\begin{array}{l}\text { This paper presents the different circuit approaches of the electric and } \\
\text { magnetic interaction of Single Split Ring Resonator (SRR) loaded microstrip } \\
\text { line. We loaded the microstrip line with planar square split ring resonator in } \\
\text { different configurations and orientations. The modeling behavior of } \\
\text { metamaterials-based microstrip lines loaded with single and two-mirrored } \\
\text { split ring resonators is analyzed numerically in two orientations (with gap of } \\
\text { SRR parallel and perpendicular to the line). The full wave simulations are } \\
\text { performed for the single and two-mirrored split ring resonators loaded } \\
\text { microstrip inside a waveguide with 'High Frequency Structure Simulator' } \\
\text { software. The equivalent circuit parameters are obtained for the single split } \\
\text { ring resonator loaded with microstrip line with the gap parallel and near to } \\
\text { the line from transmission line theory that make use of just the resonance } \\
\text { frequency and minimum of the reflection coefficient. The simulation of } \\
\text { different orientations of split ring resonator gives better reflection coefficient } \\
\text { and wider frequency. }\end{array}$} \\
\hline Received May 2, 2015 & \\
\hline Revised Aug 20, 2015 & \\
\hline Accepted Sep 4, 2015 & \\
\hline Keyword: & \\
\hline Metamaterials (MTM) & \\
\hline Microstrip line & \\
\hline Split ring resonator (SRR) & \\
\hline
\end{tabular}

Copyright (C) 2015 Institute of Advanced Engineering and Science. All rights reserved.

\section{Corresponding Author:}

Rajni,

Departement of Electronics and Communication Engineering,

Shaheed Bhagat Singh State Technical Campus,

Moga Road, Ferozepur (152004), Punjab, India.

Email: rajni_c123@yahoo.co.in

\section{INTRODUCTION}

The development of metamaterials opened a new possibility for designers to create a novel structure with unusual properties or enhanced performance [1], [2]. Metamaterials are artificially invented materials that show properties not detected in naturally occurring materials and have negative refraction index. One of the most important contributions to this topic was made in 1968 by VG Veselago who said that materials with both negative permittivity and permeability is theoretically possible [3]. In 1999, John Pendry identified a practical way to make left-handed metamaterials (LHM) which did not follow the right hand rule [4]. Later then, Smith and his colleagues demonstrated metamaterials to show negative permittivity and permeability simultaneously and carried out microwave experiments to test its unusual properties in 2000. In 2001, Smith et al showed negative refraction experimentally, using a metamaterials with repeated unit cells of split-ring resonators (SRR) and copper strips [5]-[7]. In 2002, Marques et al investigated a modified version of SRR i.e. broadside coupled (BC-SRR) to avoid bianisotropy and showed comparative analysis of the conventional (or edge-coupled) SRR and BC-SRR with printed metallic rings of the BC-SRR on both sides of the dielectric substrate [8]. In 2005, Juan Domingo Baena et al proposed new approach for designing planar metamaterial structures with SRR and complementary split-ring resonators (CSRRs) coupled to planar transmission lines and analyzed the stop band/pass band characteristics of the SRR/CSRR loaded transmission lines [9]. Bilotti et al, in 2007 discussed multiple split ring resonators (MSRR) with multiple 
rings and spiral ring (SR) to increase the miniaturized rate and concluded that increase in number of turns and rings of SRs and MSRRs respectively, increases the saturation of the resonant frequency [10]. Bojanic et al, in 2011, proposed multiband delay line with broadside coupled and the single SRRs and exhibits two lefthanded bands that can be shifted by twisting the split rings for certain angle or by changing their lengths [11]. In 2012, Sindreu et al suggested the use of SRR in coupled transmission line as compared to microwave components, to achieve better performance parameters [12]. Naqui et al in 2013 proposed a model with the electromagnetic properties of transmission lines loaded with SRRs and CSRRs randomly oriented and resonators are aligned in a non orthogonal direction to the line axis, cross-polarization effects arises[13]. Younesiraad et al in 2014 analysed resonator antenna for multi-band application with Finite Element Method and Finite Integral Technique [14]. In 2014, Kuldeep Kumar Parashar proposed a new patch antenna with compact size and large bandwidth by using simple inset feed technique [15]. In 2014, Bojanic et al presented an enhanced equivalent circuit approach for the magnetic/electric interaction of SRRs with printed lines and extract the different parameters of microstrip line with parallel and perpendicular gap to line [16].

The aim of present work is to design a microstrip line loaded with metamaterials and examine shifting of resonant frequency with different orientations of single and two mirrored SRRs. The outline of paper is as follows: Section 1 gives the brief literature review of the work done in the area. Section 2 describes the proposed SRRs loaded microstrip line model with different orientations. Section 3 presents results and discussion. Section 4 gives the conclusion of the paper.

\section{PROPOSED SRR LOADED MICRO-STRIP LINE MODEL}

In the proposed model, a conventional microstrip line is loaded with planar square SRRs with the gap parallel to the line. The square shape SRRs is coupled to microstrip line by placing it at distance' $s$ ', in the same plane. Figure 1 shows layout of SRR coupled to microstr/ip line in the same plane with gap parallel to the line. This coupled line is modeled on Rogers RO3010 substrate of thickness (h) $1.27 \mathrm{~mm}$, dielectric permittivity $\varepsilon r=10.2$ and loss tangent 0.035 . The dimensions of SRR coupled to rectangular microstrip line are given in Table 1.

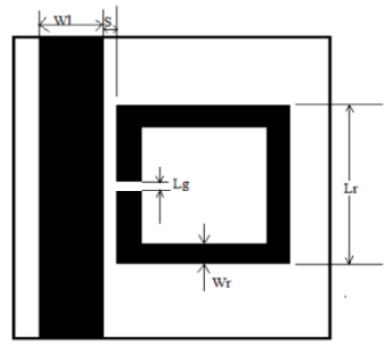

Figure 1. Layout of SRR loaded microstrip line

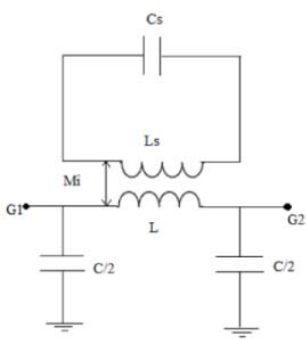

Figure 2. Equivalent circuit of SRR loaded microstrip line

Table 1. Dimensions of SRR loaded microstrip line

\begin{tabular}{cccc}
\hline S. No & Name of parameter & Representation & Dimensions(mm) \\
\hline 1 & Width of microstrip line & $\mathrm{Wl}$ & 1.2 \\
2 & Length of SRR & $\mathrm{Lr}$ & 3.0 \\
3 & Width of SRR & $\mathrm{Wr}$ & 0.2 \\
4 & Gap of split & $\mathrm{Lg}$ & 0.5 \\
5 & Gap between microstrip line \& SRR & $\mathrm{S}$ & 0.1 \\
\hline
\end{tabular}

Microstrip lines loaded with SRRs for different configurations are examined. It has been found that different configurations of SRR loaded microstrip line can be modeled by the same circuit topology, but with different values of the circuit parameters. For each topology, resonance frequency and the minimum reflection frequency can be obtained. 
1) Single SRR loaded microstrip line with the gap parallel to the line (i) gap near orientation (ii) gap far orientation

2) Single SRR loaded microstrip line with the gap perpendicular to the line

3) Two mirrored SRRs with the gap parallel to the line (i) Both gaps near orientation (ii) Both gaps far orientation

4) Two mirrored SRRs with the gap perpendicular to the line

5) Two SRRs with the gap parallel to the line (i) one gap near and one far orientation orientation

6) Two cascaded SRRs with the gap parallel to the line(i) gap near orientation (ii) gap far

7) Two cascaded SRRs with the gap perpendicular to the line

8) Double SRR loaded microstrip line with the gap parallel to the line

\subsection{SRR Loaded Microstrip Line with the Gap Parallel to the Line}

Microstrip line loaded with SRRs with gaps parallel to the line is shown in Figure 3 and 4. Figure 3 depicts the microstrip line loaded with single SRR with gap parallel to the line and gap is near to the microstrip line. In Figure 4 the gap of SRR loaded microstrip line is parallel and far from the line.

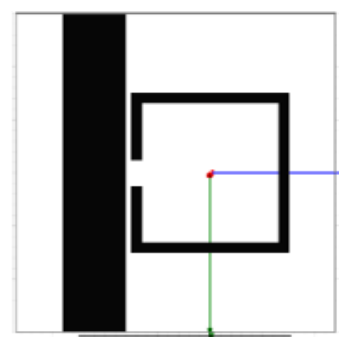

(a)

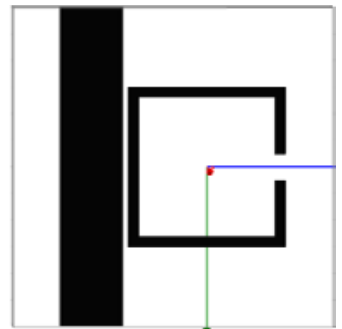

(b)

Figure 3. Microstrip line loaded with SRRs with gap parallel to the line. (a) One SRR with gap near the line (b) One SRR with the gap far from the line

To extract the parameters $\mathrm{L}$ and $\mathrm{C}$ of the transmission line in Figure 2, taking into account the coupling between the line and the nearest SRR arm, inductance is modeled as if there were two inductances.. One is coupled with the transmission line or second is isolated transmission line with length equal to the remaining uncoupled part of the SRR length. Figure 3 shows the equivalent circuit of microstrip line coupled to SRR where Ls and Cs is inductance and capacitance of SRR respectively and L and C is inductance and capacitance of microstrip line respectively. The $G_{1}$ and $G_{2}$ are two ports and $M_{i}$ is mutual inductance. The capacitance $\mathrm{C}_{\mathrm{s}}$ is obtained from the SRR resonance frequency $f_{r}$ as follows:

$$
f_{r}=\frac{1}{2 \pi \sqrt{L S C S}}
$$

Where resonance frequency is also calculated as:

$$
f_{r}=\frac{w_{r}}{2 \pi}
$$

The coupling coefficient $M_{i}$ is then obtained as a function of $f_{\text {min. }}$, the resonance frequency $f_{r}$, and the line parameters $\mathrm{L}$ and $\mathrm{C}$ as follows:

$$
M_{i}^{2}=\left(1-\frac{w_{r}^{2}}{w_{\min }^{2}}\right)\left(1-a_{1}\right)
$$

The term mutual inductance $M_{i}$ is also affected by variation of the distance ' $\mathrm{S}$ ' between the microstrip line and split ring resonator (SRR). Where $a_{1}$ correspond to the circuit with one cell and $f_{\min }=$ $\frac{w_{\min }}{2 \pi}$. These coefficients are given by:

$$
a_{1}=\left[\frac{L}{c} Y_{0}^{2}+2 b\right]
$$


Where $Y_{0}$ is the characteristic admittance of the microstrip line and:

$$
b=\left(\frac{w_{\min }}{w_{0}}\right) ; w_{0}^{2}=\frac{8}{L C}
$$

\subsection{Two Mirrored SRRs Loaded Microstrip Line with the Gap Parallel to the Line}

The microstrip line loaded with two mirrored SRRs with the gap parallel to the line is shown in Figure 4. The gap of two mirrored SRRs is near and far from the microstrip line as in Figure 4(a) and 4(b) respectively.

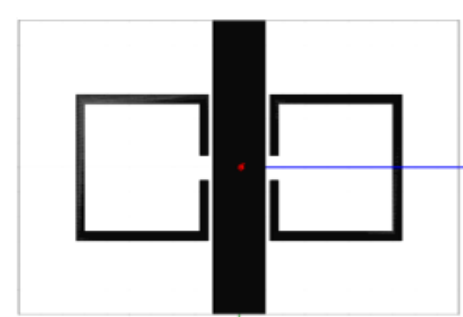

(a)

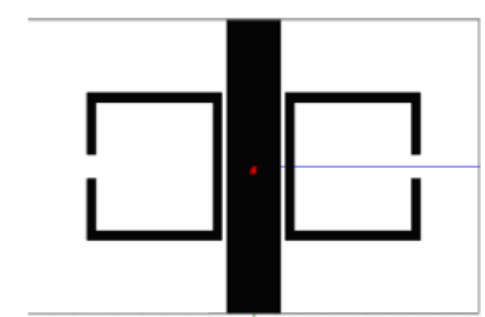

(b)

Figure 4. Microstrip line loaded with SRRs with gap parallel to the line. (a) Two SRRs with gap near the line (b) Two SRRs with the gap far from the line.

\subsection{SRRs Loaded Microstrip Line with the Gap Perpendicular to the Line}

The microstrip line loaded with single SRR and two mirrored SRRs with the gap perpendicular to the microstrip line shown in the Figure 5.

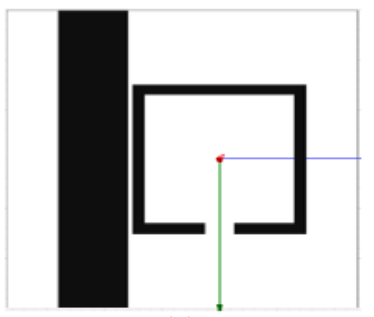

(a)

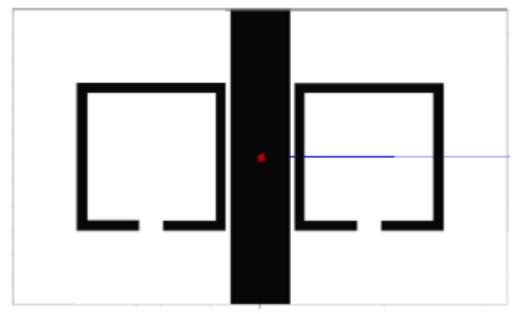

(b)

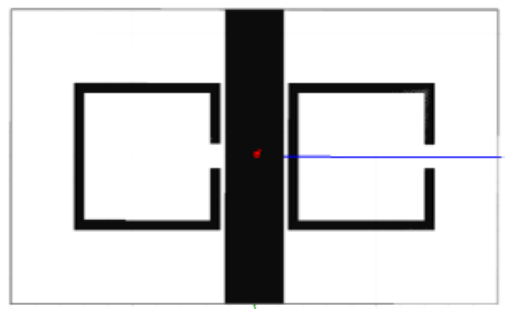

(c)

Figure 5. Microstrip line loaded with SRRs (a) Single SRR with gap perpendicular to the line (b) Two SRRs mirrored with gap perpendicular to the line (c) SRRs with one gap parallel and another perpendicular to the line

\subsection{SRRs Loaded Microstrip Line with the Gap Parallel to the Line}

The microstrip line loaded with two SRRs with the gap parallel to the line but one SRR gap has near to the line and other has far from the line as depicted in the Figure 5(c).

\subsection{Two Cascaded SRRs Loaded Microstrip Line with the Gap Parallel to the Line}

The microstrip line is loaded with two cascaded SRRs with the gap parallel to the line as seen in Figure 4. The distance'd' between the two cascaded SRRs is $0.5 \mathrm{~mm}$. The gap of two cascaded SRRs near to the microstrip line as and far from the microstrip line is shown in the Figure 6(a) and Figure 6(b) respectively. 


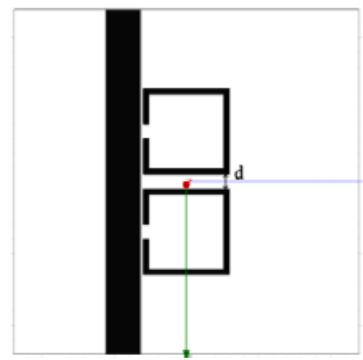

(a)

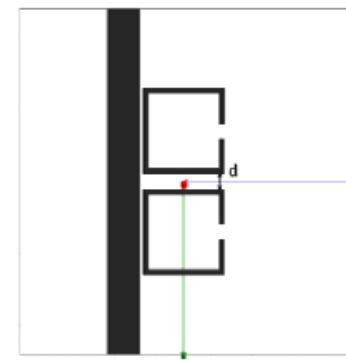

(b)

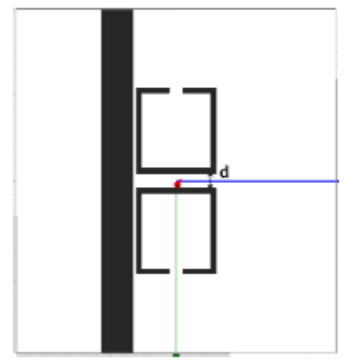

(c)

Fig. 6 Microstrip line loaded with two cascaded SRRs with. (a) Two SRRs with gap parallel and near the line (b) Two SRRs with the gap parallel and far from the line (c) gap perpendicular to the line

\subsection{Two Cascaded SRRs Loaded Microstrip Line with the Gap Perpendicular to the Line}

The microstrip line is loaded with two cascaded SRRs with the gap perpendicular to the line as seen in Figure 6(c).

\subsection{Double SRR Loaded Microstrip Line with the Gap Parallel to the Line}

Microstrip line loaded with double SRR with the gap parallel to the line is seen in Figure 7 . It depicts the microstrip line loaded with double SRR with gap parallel to the line and both splits are opposite to each other.

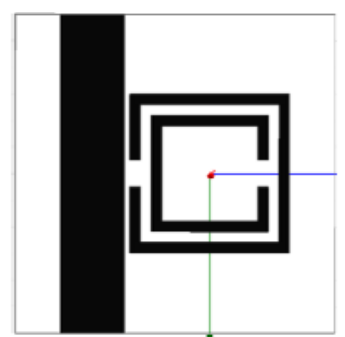

Figure 7. Microstrip line loaded Double SRR with gap parallel to the line

\section{RESULTS AND DISCUSSION}

The SRR loaded microstrip line is simulated inside a waveguide to attain the resonating frequency region. The Perfect Electric Conductor (PEC) boundary conditions are employed on the z-faces of the unit cell. The Perfect Magnetic Conductor (PMC) boundary conditions are used on top and bottom y-faces of the unit cell so that the negative permeability behavior of SRR would be excited. The two wave ports 1 and 2 are assigned to the both sides of microstrip line on the $\mathrm{x}$-faces of waveguide. The proposed structure is simulated with Ansoft software 'High Frequency Structure Simulator (HFSS)'.

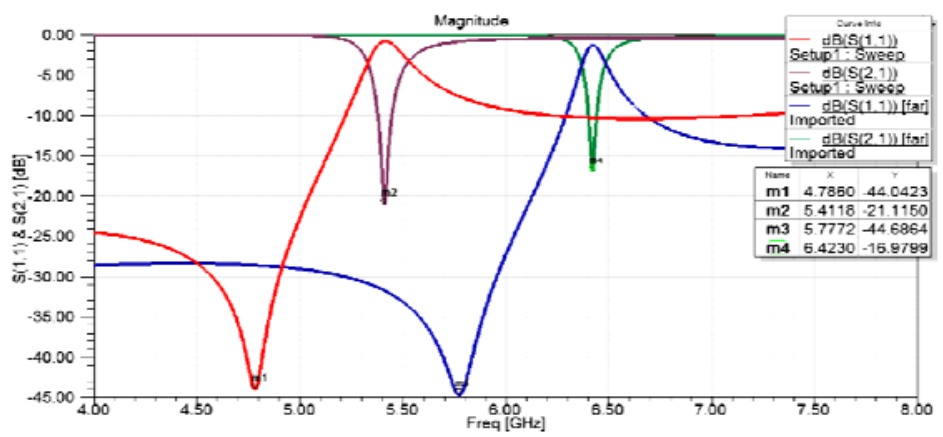

Figure 8. Reflection coefficient $S_{11}$ and Transmission coefficient $S_{21}$ of SRR loaded microstrip line with the gap parallel and near to the line and far from the line 
Figure 8 shows the reflection and transmission coefficient of SRR loaded microstrip line with the gap parallel to the line. It shows that resonant frequency is shifted to the right as the gap of SRR is changes from near to the microstrip line and far from line.

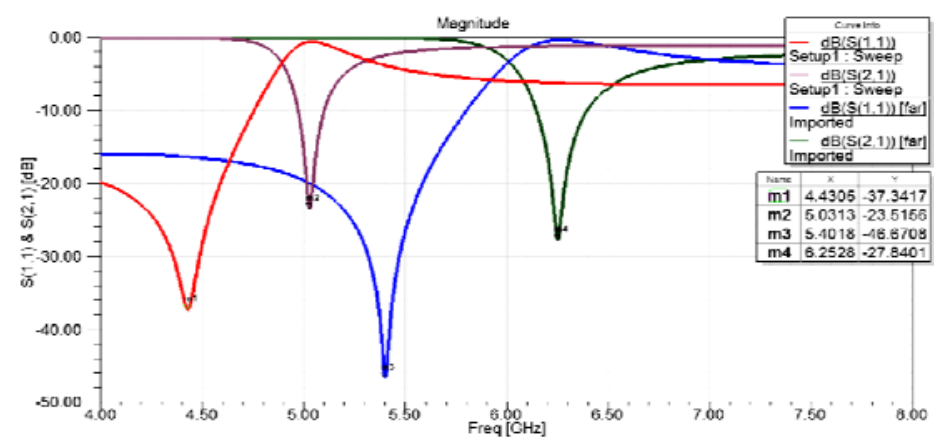

Figure 9. Reflection coefficient $S_{11}$ and Transmission coefficient $S_{21}$ of two mirrored SRRs loaded microstrip line with the gap parallel and near to the line and far from the line

Figure 9 shows the reflection and transmission coefficient of two mirrored SRRs loaded microstrip line with the gap parallel to the line. It shows that resonant frequency and return loss get increased as the gap of SRR is varied from near to far from the microstrip line.

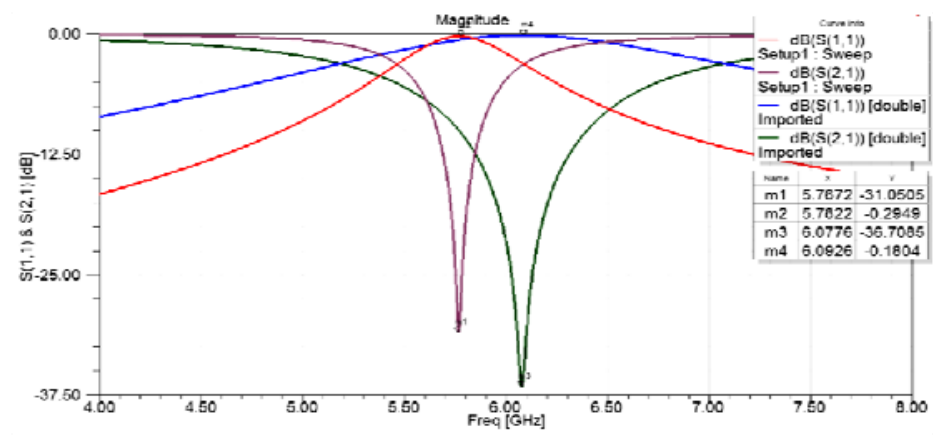

Figure 10. Reflection coefficient $S_{11}$ and Transmission coefficient $S_{21}$ of single and two mirrored SRRs loaded microstrip line with the gap perpendicular to the line

Figure 10 shows the reflection and transmission coefficient of single and two mirrored SRRs loaded microstrip line with the gap perpendicular to the line. It shows that the bandwidth and return loss is increased as the gap of SRR changes from near to the microstrip line and far from line. The resonant frequency is shifted right as the gap changes from near to the far from the line.

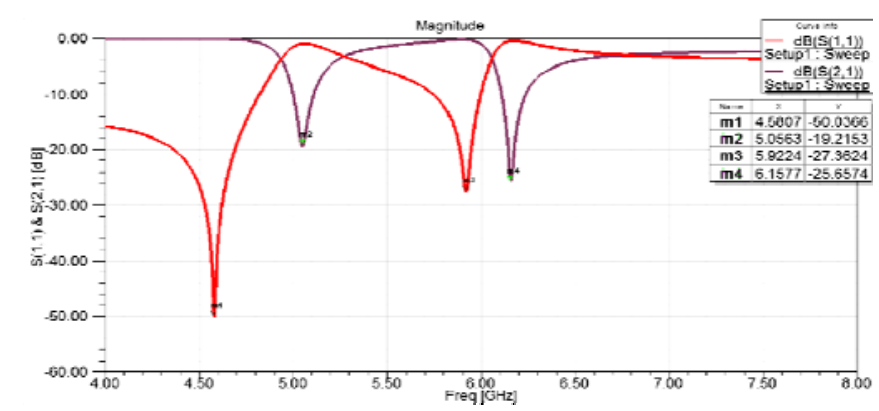

Figure 11. Reflection coefficient $S_{11}$ and Transmission coefficient $S_{21}$ of double SRRs loaded microstrip line with the gap parallel and one SRR is near and other is far from the line 
Figure 11 shows the reflection and transmission coefficient of double SRRs loaded microstrip line with the gap parallel to the line but the gap of one SRR is near to the line and other has far from the line. It shows that the bandwidth is increased and resonant frequency is shifted from lower to higher frequency region.

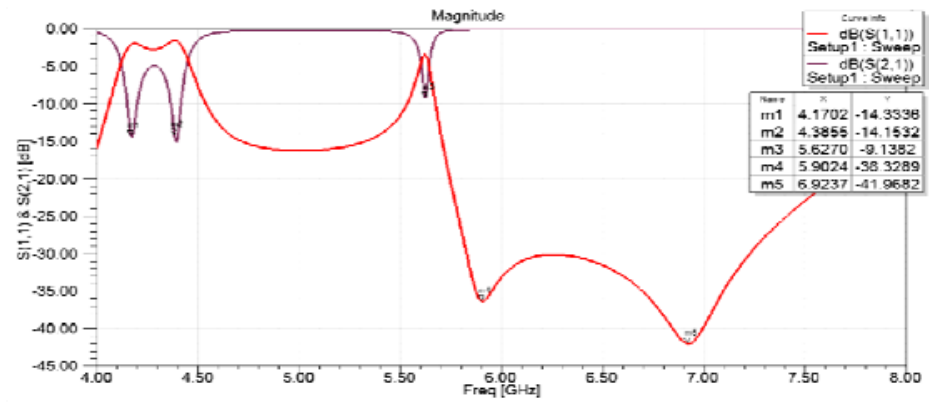

Figure 12. Reflection coefficient $S_{11}$ and Transmission coefficient $S_{21}$ of two cascaded SRRs loaded microstrip line with the gap parallel and near to the line

Figure 12 shows the reflection and transmission coefficient of two cascaded SRRs loaded microstrip line with the gap parallel and near to the line. It shows that the return loss is improved with a shift in the resonant frequency is from lower to higher region

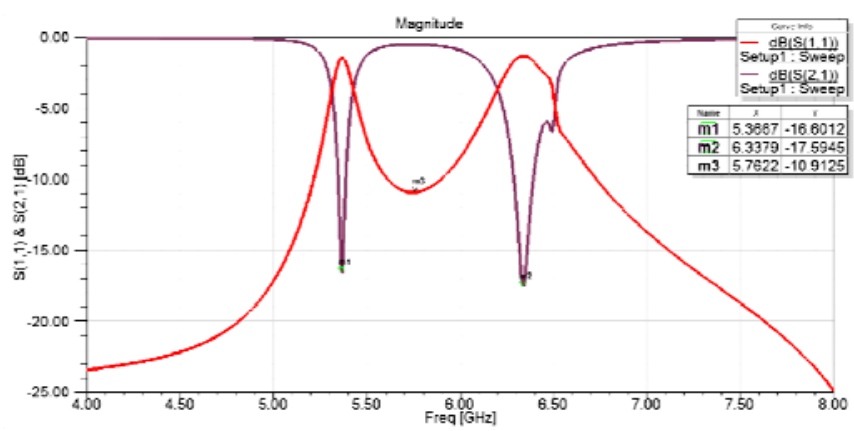

Figure 13. Reflection coefficient $S_{11}$ and Transmission coefficient $S_{21}$ of two cascaded SRRs loaded microstrip line with the gap parallel and far from the line

Figure 13 shows the reflection and transmission coefficient of two cascaded SRRs loaded microstrip line with the gap parallel and far from the line. It shows that the return loss and bandwidth is increased and resonant frequency shift to higher side.

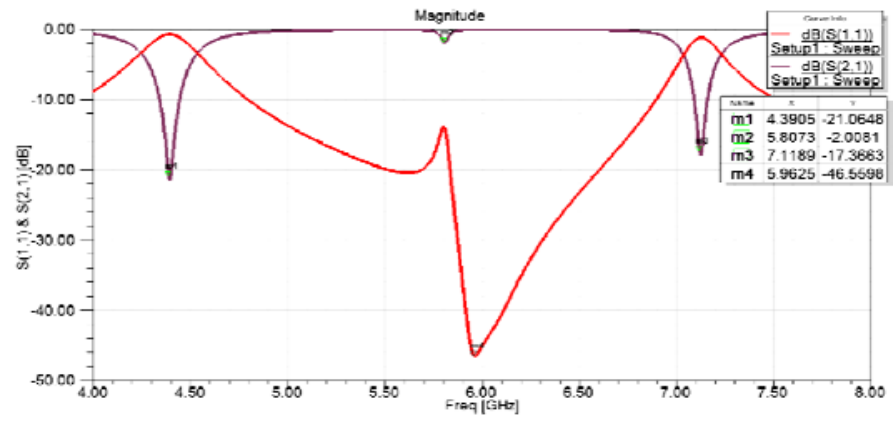

Figure 14. Reflection coefficient $S_{11}$ and Transmission coefficient $S_{21}$ of two cascaded SRRs loaded microstrip line with the gap perpendicular to the line 
Figure 14 shows the reflection and transmission coefficient of two cascaded SRRs loaded microstrip line with the gap perpendicular to the line. It can be seen that resonant frequency is shifted to higher side.

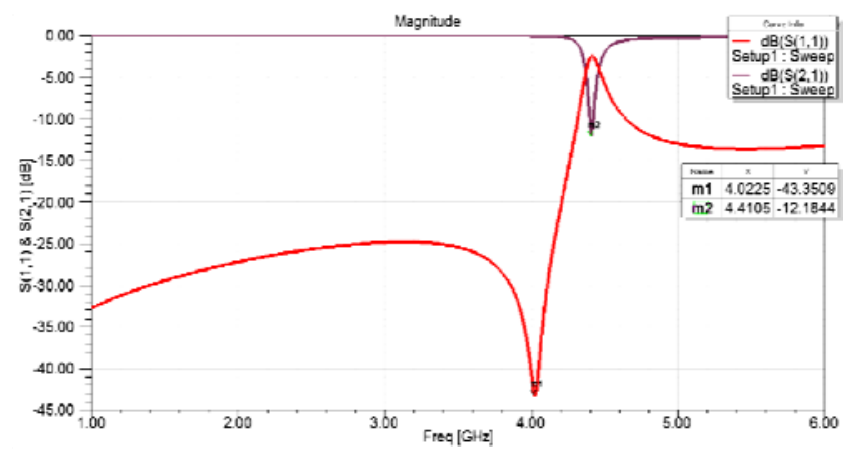

Figure 15. Reflection coefficient $S_{11}$ and Transmission coefficient $S_{21}$ of double SRR loaded microstrip line with the gap parallel to the line

Figure 15 shows the reflection and transmission coefficient of double SRR loaded microstrip line with the gap parallel to the line. It shows that the transmission line resonates at lower frequency as compared to all other compared to all other orientations.

\section{CONCLUSION}

In this paper, microstrip lines loaded with single and double split-ring resonators in different orientations have been proposed. These orientations of the SRR with respect to the line are analyzed with the parallel gap near and far from the line, with the gap perpendicular to the line. The printed line is loaded with a single SRR at one side, or with two mirrored SRRs placed with respect to the line. This type of structures demonstrates stop band response, but the proposed equivalent circuit model can easily be extended to structures with pass band response. The improved equivalent circuit of proposed SRR model moves the resonance to higher frequencies. The cause of frequency shifting is variation in capacitance of strip line due to SRRs coupled in various configurations. Therefore, the bandwidth is increased along with good matching.

\section{REFERENCES}

[1] G. V. Eleftheriades and K. G. Balmain, Eds., "Negative- Refraction Metamaterials: Funda-mental Principles and Applications", New York: Wiley, 2005.

[2] B. A. Munk, Metamaterials: Critique and Alternatives. New York: Wiley, 2009.

[3] V. G. Veselago, "The electrodynamics of substances with simultaneously negative values of " $\varepsilon$, and $\mu$ ", Sov. Phys.-Usp.,Vol. 47, pp. 509-514, 1968.

[4] J. P. Pendry, A. J. Holden, D. J. Robbins, W. J. Stewart, IEEE. Transmission Microwave Theory Technology, Vol. 47, pp. 2075, 1999.

[5] R. A. Shelby, D. R. Smith, S. Schultz, Refraction, Science Magazine, 6, Vol. 292, pp. 5514, 2001

[6] D. R. Smith, W. J. Padilla, D. C.Vier, S.C. Nemat-Nasser, and S. Schultz, "Composite medium with simultaneously negative permeability and permittivity”, Phys. Rev. Lett., Vol. 84, pp. 4184-4187, 2000.

[7] Smith, David R., "What are Electromagnetic Metamaterials?", Novel Electromagnetic Materials., The research group of D.R. Smith, 2009.

[8] Ricardo Marqués, Francisco Mesa,, Jesús Martel, and Francisco Medina, "Comparative Analysis of Edge- and Broadside-Coupled Split Ring Resonators for Metamaterial Design-Theory and Experiments", IEEE Transaction on Antennas and Propagation, Vol. 51, No. 10, 2003.

[9] Juan Domingo Baena, Jordi Bonache, Ferran Martín, Ricardo Marqués Sillero, Francisco Falcone, Txema Lopetegi, Miguel A. G. Laso, Joan García-García, Ignacio Gil, Maria Flores Portillo, and Mario Sorolla, “Equivalent-Circuit Models for Split-Ring Resonators and Complementary Split-Ring Resonators Coupled to Planar Transmission Lines", IEEE Transactions On Microwave Theory and Techniques, Vol. 53, No. 4, 2005.

[10] Filiberto Bilotti, Alessandro Toscano, and Lucio Vegni, "Design of Spiral and Multiple Split-Ring Resonators for the Realization of Miniaturized Metamaterial Samples", IEEE Transactions On Antennas and Propagation, Vol. 55, No. 8, 2007.

[11] Radovan Bojanic, Branka Jokanovic, and Vojislav Milosevic, "Reconfigurable Delay Lines with Split-Ring Resonators", Microwave Review,2011. 
[12] Miguel Durán-Sindreu, Jordi Naqui, Ferran Paredes, Jordi Bonache, and Ferran Martín, "Electrically Small Resonators for Planar Metamaterial, Microwave Circuit and Antenna Design: A Comparative Analysis", Appl. Sci., Vol. 2, pp. 375-395, 2012.

[13] Jordi Naqui, Miguel Durán-Sindreu and Ferran Martín, "Modeling Split-Ring Resonator (SRR) and Complementary Split-Ring Resonator (CSRR) Loaded Transmission Lines Exhibiting Cross-Polarization Effects", IEEE Antennas and Wireless Propagation Letters, Vol. 12, 2013.

[14] Hemn Younesiraad, Mohammad Bemani, Saied Nikmehr, "Small Multi-Band Rectangular Dielectric Resonator Antennas for Personal Communication Devices", International Journal of Electrical and Computer Engineering, Vol. 4, No.1, pp. 1-6, 2014

[15] Kuldeep Kumar Parashar, "Design and Analysis of I-Slotted Rectangular Microstrip Patch Antenna for Wireless Application", International Journal of Electrical and Computer Engineering, Vol. 4, No.1, pp. 31-36, 2014.

[16] Radovan Bojanic, Vojislav Milosevic, Branka Jokanovic, Francisco Medina-Mena, and Francisco Mesa, "Enhanced Modelling of Split-Ring Resonators Couplings in Printed Circuits", IEEE Transactions on Microwave Theory and Techniques, Vol. 62, No. 8, 2014.

\section{BIOGRAPHIES OF AUTHORS}
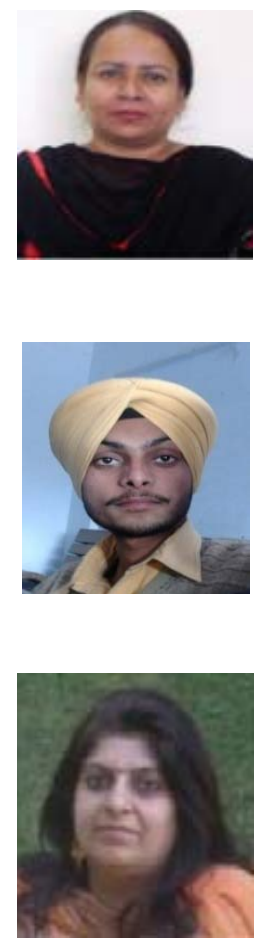

Rajni is currently Associate Professor at SBS StateTechnical Campus Ferozepur, India. She has completed her M.E. from NITTTR, Chandigarh, India and B.Tech from NIT, Kurukshetra India. She is pursuing her Ph.D. in metamaterial antennas. She has approx. 17 years of academic experience. She has authored a number of research papers in International journals, National and International conferences. Her areas of interest include Wireless communication and Antenna design.

Gurwinder Singh is currently pursuing M.Tech from SBS State Technical Campus, Ferozepur, India. He has completed B.Tech from PTU Jalandhar in 2012. His areas of interest include Antenna design.

Dr Anupma Marwaha is currently Associate Professor at Sant Longowal Institute of Engg. \& Tech, Logowal (Sangrur). She has done her Ph.D from GNDU, Amritsar, M. Tech. from REC Kurukshetra (Now NIT, Kurukshetra), B.E from Punjab University,Chandigarh.She has 22 years of academic experiemce. She has authored 25 research papers in International and National Journals and 50research papers in National and International conferences. She has supervised 02 $\mathrm{Ph} . \mathrm{D}$ Thesis and $10 \mathrm{M}$.Tech thesis and 04 are under progress.Her areas of interst include Electromagnetics,Microwave Comm,Wireless communication and Antenna Design 